\title{
Gradual reduction of benzodiazepines, with or without cognitive behavioural therapy, increases successful withdrawal rates compared with no support in long-term users
}

\author{
Voshaar R, Gorgels W, Mol A et al. Tapering off long-term benzodiazepine use with or without group \\ cognitive-behavioural therapy: three condition, randomised controlled trial. Br J Psychiatry 2003; 182:498-504.
}

QUESTION: In a primary care setting, does a tapering schedule, with or without group cognitive behavioural therapy, help to ensure successful withdrawal from benzodiazepines among long-term users?

\section{Design \\ Randomised controlled trial. \\ Setting \\ 30 general practices, The Netherlands.}

\section{Participants}

180 people unable to withdraw from long term $(\geq 3$ months) benzodiazepine use by themselves. Exclusion criteria were: a history of psychosis, epilepsy, terminal illness, a severe comorbidity or psychosocial problems, current psychiatric treatment, or current treatment for alcohol or drug dependence. People who were unable to speak Dutch were also excluded.

\section{Intervention}

People were randomised to 3 groups: tapering of medication only; tapering of medication plus group cognitive behavioural therapy over 3 months, or no support. The tapering process involved converting all the participants' medication to the equivalent dosage of diazepam and then reducing the diazepam dosage by $25 \%$ per week for 4 weeks. In the last week, participants could choose to reduce the last dose in 2 steps of $12.5 \%$ at 4 day intervals instead of the full 25\%. The group therapy consisted of 2 hour cognitive behaviour therapy sessions each week for 5 weeks, starting halfway through the withdrawal programme. The sessions included education about benzodiazepines and advantages of stopping therapy, relaxation techniques, and cognitive restructuring to alter participants' interpretation of withdrawal symptoms.

\section{Main outcome measures}

The primary outcome was successful withdrawal from medication, assessed by participants' self reports, checked against their general practitioner's prescription database.

\section{Main results}

Tapering, with or without cognitive behavioural therapy, significantly increased successful withdrawal at 2 months compared with no support (successful withdrawal: $45 \%$ with tapering plus cognitive behavioural therapy; $51 \%$ with tapering alone; $15 \%$ with no support; $\mathrm{p}=0.002$ for each intervention compared with no support). However, adding cognitive behavioural therapy did not significantly improve withdrawal rates compared with tapering alone.

\section{Conclusions}

The tapering intervention increases benzodiazepine withdrawal rates in primary care settings. Group cognitive behavioural therapy does not improve upon rates achieved with the tapering schedule alone.

\section{COMMENTARY}

The clinical management of benzodiazepine withdrawal is difficult and there is little evidence to guide clinicians on the best way to do this. This study is therefore welcomed as it is one of the few population based studies aimed at evaluating drug tapering (a widely used strategy ${ }^{1-3}$ ) and adjunctive group cognitive behavioural therapy. A problem with such studies is recruitment; this study is no exception with $81 \%$ of eligible patients refusing to participate. Despite this, the final sample size with 180 patients being randomised was sufficiently powered to detect differences between the groups. This final sample is biased as they may have been more motivated to withdraw from their benzodiazepines and patients who were in psychiatric treatment or who had drug or alcohol dependence were excluded.

The main outcome measure of benzodiazepine discontinuation was taken 3 months after the start of the intervention; a longer follow-up would have been helpful to ensure there was no relapse.

Nonetheless, despite these methodological limitations, this study provides guidance for the clinician and suggests using a long acting benzodiazepine and tapering $25 \%$ of the dose per week (and weekly consultations) is more effective than just suggesting withdrawal (NNT=3). The lack of effect of cognitive behavioural therapy may be the result of using group rather than individual treatment, which might have been more appropriate. However, this study does not help with the issue of withdrawing benzodiazepines for patients with a comorbid psychiatric disorder when more extensive cognitive behavioural therapy may be of more help. ${ }^{4}$ As part of the recruitment process for this study the participating general practitioners wrote to all of their patients who had been on long term benzodiazepines advising them to quit; $14 \%$ of the patients did so before randomisation. This is a useful first step in the management of benzodiazepine dependence and will help some patients decide to stop taking their benzodiazepines.

Professor Philip Boyce, MD FRANZCP Department of Psychological medicine University of Sydney Nepean Hospital Penrith, Australia

1 Spiegel DA. Psychological strategies for discontinuing benzodiazepine treatment. J Clin Psychopharmacol 1999;19: $17 \mathrm{~S}-22 \mathrm{~S}$.

2 Kosten TR, O'Connor PG. Management of drug and alcohol withdrawal. N Engl J Med 2003;348:1786-95.

Schweizer E, Rickels K, Case G, Greenblatt DL. Long-term therapeutic use of benzodiazepines: II. Effects of gradual taper. Arch Gen Psychiatry 1990;47:908-915.

4 Otto MW, Pollack MH, Sachs GS et al. Discontinuation of benzodiazepine treatment: efficacy of cognitive-behavioral therapy for patients with panic disorder. Am J Psychiatry 1993; 150: 1485-1490.
Source of funding:

The study was funded by the Dutch Health Care Insurance Council.

For correspondence: $R$ Voshaar. University Medical Center St Radboud, Department of Psychiatry (hp333) P.O. Box 9101,6500 HB Nijmegen, The Netherlands. r.oudevoshaar@ psy.umcn.nl 